Particle physics

\section{Can the gallium detector solve the solar neutrino problem?}

\section{from W. Hampel}

SOLAR neutrino experiments, designed to detect the neutrinos generated in the nuclear fusion processes in the Sun, are among those difficult experiments that aim to detect very rare events. The only type of detector able to overcome the huge background problems in detecting these neutrinos is the radiochemical detector. An important quantity for radiochemical experiments is the neutrino capture crosssection for the target nucleus. A recent paper of Orihara et al. ${ }^{1}$ is concerned with the cross-section of the gallium detector, upon which rest current hopes of resolving the discrepancy known as the solar neutrino problem.

The only completed experimental attempt to detect solar neutrinos, the Brookhaven Chlorine Experiment ${ }^{2}$, has resulted in a neutrino signal of $1.8 \pm 0.3 \mathrm{SNU}$ $(1 \mathrm{SNU}=1$ neutrino capture per second in $10^{36}$ target atoms). This is almost four times lower than the $6.9 \mathrm{SNU}$ predicted by the so-called Standard Solar Model (SSM) ${ }^{3.4}$. It is this discrepancy that has long been known as the solar neutrino problem.

Because of the energy threshold (814 $\mathrm{keV})$ of the ${ }^{37} \mathrm{Cl}\left(\nu, \mathrm{e}^{-}\right)^{37} \mathrm{Ar}$ reaction on which the chlorine detector is based, it is mainly sensitive to the ${ }^{7} \mathrm{Be}(862 \mathrm{keV})$ and ${ }^{8} \mathrm{~B}$ (0-14 MeV) neutrinos. While contributing 75 per cent of the expected rate in this experiment, ${ }^{8} \mathrm{~B}$ neutrinos are produced in a very rare side branch of the solar fusion chain and are insignificant in the total energy production in the Sun. Unfortunately, the chlorine detector cannot respond to the bulk of solar neutrinos, the pp neutrinos $(0-420 \mathrm{keV})$, generated in the primary fusion reaction.

It has long been recognized that an experiment with a threshold low enough to detect the pp neutrinos might, among other things, provide the answer to the problem imposed by the chlorine experiment. The only experiment of this type which has been demonstrated to be feasible is the gallium experiment ${ }^{5}$, based on the neutrino capture reaction ${ }^{71} \mathrm{Ga}\left(\nu, \mathrm{e}^{-}\right)^{71} \mathrm{Ge}(236 \mathrm{keV}$ threshold). The SSM prediction for that experiment $^{3}$ is 106 SNU (pp: 66 per cent, ${ }^{7} \mathrm{Be}: 27$ per cent). Even if one assumes that the low chlorine result is due to some unknown problems with the solar model, the most probable result for the gallium detector will still be near $85 \mathrm{SNU}$.

The only other seriously discussed explanation for the solar neutrino problem is a reduced electron neutrino flux at the detector due to neutrino oscillations; mixing of the electron neutrino with neutrinos of other flavours (oscillations) may occur if neutrinos are not mass eigen- states. In this case the expected neutrino signal for the gallium detector would be distinctly reduced and certainly less than 70 SNU. That is why the gallium detector should be able to decide whether the explanation of the solar neutrino problem is that there are serious problems with the solar model or that neutrinos oscillate. The latter would involve a non-zero rest mass of the neutrino, which would have severe consequences for elementary particle physics and, possibly, for cosmology.

The interpretation of the result from a gallium detector requires knowledge of the cross-section for neutrino capture in ${ }^{71} \mathrm{Ga}$. This cross-section is dominated by transitions to the ${ }^{71} \mathrm{Ge}$ ground state, which can be reliably calculated from the observed $\beta$-decay between the two states ${ }^{6}$. The rates given above for the gallium detector are based on this ground state cross-section. However, there may be additional contributions from two states in ${ }^{71} \mathrm{Ge}$ at excitation energies 175 and $500 \mathrm{keV}$ which may be populated in particular by ${ }^{7} \mathrm{Be}$ neutrinos. In order to calculate the cross-section leading to excited states one needs the $\beta$-decay ft-values (Gamow-Teller strength) of the respective states. In principle this information can be obtained from the systematics of $\beta$-decay or from $(p, n)$ forward scattering experiments since the $(p, n)$ charge-exchange reaction connects the same states in the target and product nuclei as neutrino capture or (in inverse direction) $\beta$-decay.

Orihara and co-workers ${ }^{1}$ have measured the ${ }^{71} \mathrm{Ga}(\mathrm{p}, \mathrm{n})$ reaction at $35 \mathrm{MeV}$ proton energy and have extracted from their data ft-values corresponding to transitions from the ${ }^{71} \mathrm{Ga}$ ground state to the ${ }^{71} \mathrm{Ge}$ states at 175 and $500 \mathrm{keV}$. Their results yield a total rate of $135 \mathrm{SNU}$ for the gallium detector, 29 SNU larger than the predicted capture rate for the transition to the ground state alone (the number of $149.6 \mathrm{SNU}$ quoted in their
The Remarkabie Sunsets

THOUGH we are no longer favoured with the gorgeous sunsets which marked the autumn and early winter, yet two phenomena are still frequently visible which seem referable to the same cause as those splendid displays.

The first is the unusual white glow in the western sky before sunset which was an almost constant precursor of the brilliant and longcontinued colouring of the past months. It was very marked on November 8 , the occasion of the first remarkable sunset, and it still to be seen on almost any fine evening before the sun sets, though it is no longer followed by the more striking phenomena.

The second is a decidedly unusual pink tinge paper is incorrect because of a computational error). The increase is dominated by an increased ${ }^{7} \mathrm{Be}$ fraction in this rate (pp: 53 per cent, ${ }^{7} \mathrm{Be}: 36$ per cent). This causes the prediction for the gallium detector to become more dependent on details of the solar model. It will not, however, qualitatively affect the potential of the gallium experiment to provide the answer to the solar neutrino problem.

In addition, it should be noted that there is a problem connected with the interpretation of the $(p, n)$ data at $35 \mathrm{MeV}$ in terms of $\beta$-decay ft-values: there could be contributions to the $(p, n)$ cross-section which do not simply correspond to allowed GamowTeller transitions. Consequently, the neutrino capture cross-section extracted from $(p, n)$ measurements would be overestimated. Indications that this may be the case come from a preliminary result of 14 SNU for the excited state contribution in a $(p, n)$ experiment on ${ }^{71} \mathrm{Ga}$ using $120 \mathrm{MeV}$ protons at the Indiana University cyclotron (J.N. Bahcall, personal communication).

Since there will always remain some ambiguities in the interpretation of $(p, n)$ data in terms of neutrino capture crosssection, it is important to note that an experiment with an artificial ${ }^{51} \mathrm{Cr}$ neutrino source planned as an integral part of the gallium experiment ${ }^{5}$ will eventually be able to answer the question about excited state contributions. ${ }^{51} \mathrm{Cr}$ emits neutrinos of 746 $\mathrm{keV}$, sufficient in energy to populate the 175 and $500 \mathrm{keV}$ levels in ${ }^{71} \mathrm{Ge}$. The ${ }^{51} \mathrm{Cr}$ experiment will thus provide not only a test of the entire detector system but will also yield direct information on the ${ }^{71} \mathrm{Ga}$ neutrino capture cross-section relevant for solar neutrinos.

W. Hampel is at the Max Planck Institute for Nuclear Physics, D-6900 Heidelberg I, Postfach 103980 , Saupfercheckweg 1, FRG.

\footnotetext{
Orihara, H. el al. Phys. Rev. Letl. 51, 1328 (1983).

Davis, R., Cleveland, B.T. \& Rowley, J.K. Science Underground AIP Conf. Proc \%, 2 (1983).

Bahcall, I. N. Huebner, W.F. L ubow, S.H., Parker, P.D. \& Ulrich, R.K. Rev. Mod. Phys. 54, 767 (1982)

4. Fowler, W.A. Science Underground, AIP Conf. Proc. 9. 80 (1983).

. Hampel, W. Science Underground, AIP Conf. Proc. 96, 88 (1983).
}

6. Bahcall, J.N. Rev. Mod. Phys. 50, 881 (1978).

\title{
100 years ago
}

occasionally visible for some ten to twenty degrees round the sun when shining in a somewhat hazy sky, the colour being brought out with great distinctness if light cumulus cloud happens to be passing accross it. I first observed it about 1 p.m. on Sunday, March 2, and it was very marked last Thursday (20ti1) between 10 and 11 a.m., and again on Friday ( $21 \mathrm{st}$ ) between 1 and 2 p.m., as well as on one or two other days which 1 have not specially noted.

May not both be due to the gradual subsidence to a lower level in our atmosphere of the particles which at a higher elevation caused the wonderful colouring of the past months?

Hampstead, March 24 B. W.S. 